Research article

\title{
A candidate metastasis-associated DNA marker for ductal mammary carcinoma
}

\author{
Patnala Mohan R Achary ${ }^{1}$, Hui Zhao ${ }^{1}$, Zuoheng Fan ${ }^{1}$, Swarna Gogineni², Venkat R Pulijaal², \\ Lawrence Herbst ${ }^{3}$, Panna S Mahadevia ${ }^{3}$, Joan G Jones ${ }^{3}$, Harold P Klinger ${ }^{4}$ \\ and Bhadrasain Vikram ${ }^{1}$
}

\author{
${ }^{1}$ Metastasis Laboratory, Department of Radiation Oncology, Albert Einstein College of Medicine and Montefiore Medical Center, Bronx, New York, \\ USA \\ ${ }^{2}$ Department of Obstetrics and Gynecology, Albert Einstein College of Medicine and Montefiore Medical Center, Bronx, New York, USA \\ ${ }^{3}$ Department of Pathology, Albert Einstein College of Medicine and Montefiore Medical Center, Bronx, New York, USA \\ ${ }^{4}$ Department of Molecular Genetics, Albert Einstein College of Medicine, Bronx, New York, USA \\ Corresponding author: Patnala Mohan R Achary (e-mail: achary@aecom.yu.edu)
}

Received: 8 October 2002 Revisions received: 4 December 2002 Accepted: 17 December 2002 Published: 30 January 2003

Breast Cancer Res 2003, 5:R52-R58 (DOI 10.1186/bcr571)

(C) 2003 Achary et al., licensee BioMed Central Ltd (Print ISSN 1465-5411; Online ISSN 1465-542X). This is an Open Access article: verbatim copying and redistribution of this article are permitted in all media for any non-commercial purpose, provided this notice is preserved along with the article's original URL.

\begin{abstract}
Background: Molecular genetic markers to identify the $13 \%$ lymph node-negative mammary carcinomas that are prone to develop metastases would clearly be of considerable value in indicating those cases in need of early aggressive therapy.

Methods: Representational difference analysis was used in an attempt to identify genetic alterations related to breast cancer metastasis by comparing genomic DNA from microdissected normal cells and from metastatic cells of ductal breast carcinoma patients.
\end{abstract}

Results: Representational difference analysis products yielded 10 unique metastasis-associated DNA sequences (MADS), i.e. products apparently lost in metastatic cell DNA. Of these sequences, MADS-IX was found to be lost in the transition from primary to metastasis in two out of five ductal breast carcinoma cases. This sequence was localized on chromosome $10 q 21$ by radiation hybrid mapping and fluorescence in situ hybridization. The PTEN gene, which is also located on chromosome 10q, was detected to be present by PCR in all five cases. On the contrary, a breast carcinoma cell line, HCC-1937, which has homozygous loss of a region encompassing the PTEN gene, showed the presence of MADS-IX. PCR screening of three additional breast carcinoma cell lines with known losses in specific chromosomal regions also showed the presence of MADS-IX.

Conclusion: These data suggest that MADS-IX possibly is part of a novel candidate metastasis-associated gene located close to the PTEN gene on chromosome 10q. The first set of PCR screening in five patient samples indicates that it could be used as a molecular marker for ductal mammary metastasis.

Keywords: archival tumor samples, mammary carcinoma, metastasis, molecular markers, representational difference analysis

\section{Introduction}

The clinical outcome is generally positive for patients with node-negative breast carcinoma (i.e. those patients without detectable metastases in the lymph nodes) who have been treated with surgery or with surgery and radiation therapy. The disease spreads in about $13 \%$ of the patients, however, and their lives are at risk of death [1-3]. Genetic tests capable of identifying patients at risk for metastatic spread and/or better treatment targeted to eradicate metastatic tumor deposits could have a dramatic impact on the overall survival of these patients.

The construction of a panel of molecular genetic markers for identifying the $13 \%$ lymph node-negative mammary carcinomas that are prone to develop metastases [3] would be valuable in indicating those cases in need of early

$\mathrm{bp}=$ base pairs; $\mathrm{FISH}=$ fluorescence in situ hybridization; $\mathrm{LCM}=$ laser capture microdissection; MADS = metastasis-associated DNA sequences; 
aggressive therapy. There would also be considerable benefit to the $87 \%$ of women with mammary carcinomas who are not likely to metastasize by sparing them the physical, mental and financial costs of the treatment. In addition, further knowledge of the genetic mechanisms that play an important role in metastasis could ultimately lead to the development of improved therapeutic procedures.

The evidence for the role of gene alterations in promoting metastasis in general, and in mammary carcinomas in particular, is still accumulating. It is probable, however, that like tumorigenicity suppressor genes and oncogenes many more genes involved in metastasis remain to be discovered since the process is complex, involving a large number of pathways [4]. Most of the known genetic mechanisms involved in the progression of a tumor to the metastatic state involve the loss of function of genes that prevent cells from becoming invasive. These are similar to tumorigenicity suppressor genes, whose loss of wild-type growth regulatory function leads to unregulated or malignant growth. Similarly, the wild-type metastasis suppressor genes regulate the cell's mobility and its response to environmental messages, presumably keeping most cells localized and differentiated. Mutation or complete loss (deletion) of the wild-type counterpart of any such gene in a malignant cell may make that cell more motile, invasive or metastatic. In particular, mutations of genes regulating cell adhesion molecules have been reported to be metastasis-fostering alterations [5-8]. In human breast cancers, several other genes have been found to be associated with metastasis, namely $n m 23$ [9,10], KA/1 [11,12], mta1 [13], KiSS1 [14], PTEN/MMAC1 [15,16] and BRMS1 [17].

Representational difference analysis (RDA), a DNA subtractive hybridization method [18], was used in the present study to test the working hypothesis that, to achieve the metastatic state, primary mammary carcinoma cells must acquire genetic changes in addition to those changes that led to transformation. With the RDA method, DNA from normal cells was compared with that of the metastatic cells of the same patient. The recovered differential sequences were then mapped on human chromosomes and used to screen DNA samples from normal, primary and metastatic cells of five additional ductal mammary carcinoma patients to determine whether these sequences were consistently associated with metastasis.

\section{Materials and methods} Tissue samples and cell lines

Normal breast tissue and lymph nodes with metastatic cells of a 52-year-old patient (C-1050) with ductal mammary carcinoma were provided by the Cooperative Human Tissue Network. Additional matched normal, primary and metastatic tissue samples were collected from four patients (patient 1, C-18805; patient 2, C-98-05H; patient 3, C-19898; and patient 4, C-20635) from the Cooperative Human Tissue Network and from one patient (patient 5, DS-9605) from the Surgical Pathology Department of Albert Einstein College of Medicine and Montefiore Medical Center. The DNA samples from mammary carcinoma cell lines (HCC-1806, HCC-11433, HCC-1428 and HCC-1937) and matched normal cell lines were received from Dr R Parson of Columbia University, New York, USA.

\section{Isolation of cells from biopsy samples by single cell microdissection}

Single cell microdissection (SCM) was performed on hematoxylin and eosin-stained tissue sections of positive lymph nodes from the ductal breast cancer patient samples [19]. In the present method of SCM, a Zeiss axiovert phase contrast photomicroscope fitted with a television monitor and a Narashige mechanical microdissector was used. A glass micropipette was used to draw up individual cells after dissection.

Tumor cells were identified in the microscope and, using the tip of a glass micropipette attached to a syringe, they were dissected out without disturbing the surrounding tissue and were then drawn into the pipette. After five to 10 cells were collected in the tip, it was broken off and dropped into a sterile eppendorf tube. Approximately 10,000 metastatic tumor cells were collected in this manner for RDA experiments. The SCM method was used to isolate tumor cells for the RDA experiment because laser-capture microdissection (LCM) equipment was initially not available.

\section{Isolation of cells from biopsy samples by LCM}

LCM [20] was used for isolating tumor cells from primary and metastatic tissue samples of additional patients for screening candidate metastasis-associated DNA sequences (MADS) by PCR. After many tumor cells have been captured on the LCM cap, it is then placed on a $500 \mu \mathrm{l} \mathrm{PCR}$ tube containing ATL lysis buffer (Qiagen Inc, Valencia, CA, USA) and the DNA is extracted.

\section{DNA extraction}

About $1 \mu \mathrm{g}$ DNA was isolated from approximately 10,000 cells microdissected from metastatic tumor and normal (glandular and stromal) breast tissue samples, using a modified method of DNA extraction from archival tissues. Briefly, the microdissected cell pellet was incubated overnight in lysis buffer (Qiagen Inc) with proteinase- $\mathrm{K}$ at $55^{\circ} \mathrm{C}$. Glycogen (carrier) was added to the cell lysate and DNA was extracted using a phenol-chloroform-isoamyl alcohol mixture followed by ethanol precipitation [19].

\section{RDA and characterization of differential products}

The RDA procedure was basically as described by Lisitsyn and coworkers $[18,21]$ using the $B g / l$ representation. The metastatic cancer cell DNA was used as the 'driver' 
and the DNA from the normal cells was the 'tester'. Three rounds of hybridization were performed to subtract the common sequences. The primers used for representation and hybridization steps of RDA are presented in Table 1. DNA from the differential bands (lost in metastasis) was cloned using the TA cloning system (pCR2.1; Invitrogen Co, Carlsbad, CA, USA). Clones were selected at random and used as $\alpha^{32} \mathrm{P}$-dCTP (Amersham Biosciences Corp, Piscataway, NY, USA) probes on dot blots or Southern blots containing amplicon DNA from the original normal and metastatic cells to verify whether these sequences are actually present in the normal cell DNA sample and missing in the metastatic cell DNA samples. Positive clones (showing signals in normal cell DNA and missing in metastatic cell DNA) were sequenced. A homology search was performed using the National Center for Biotechnology Information, National Institutes for Health, USA (NCBI) Blast program and physical mapping.

\section{Radiation hybrid mapping}

Radiation hybrid mapping was used to localize the MADS on human chromosomes using high-resolution GeneBridge 4 radiation hybrid panels (Research Genetics, Inc, Carlsbad, CA, USA). Based on the sequences of differential products isolated from RDA experiments, 10 pairs of PCR primers were designed for 10 groups of candidate gene sequences. Based on the PCR results, chromosome localization of these sequences was determined using the Whitehead Institute/MIT databases [22].

\section{Fluorescence in situ hybridization of MADS-IX}

Chromosome preparations were made from phytohemagglutinin-stimulated peripheral blood lymphocyte cultures from normal individuals. The slides were fixed in methanol-acetic acid and were air-dried. The probes used for fluorescence in situ hybridization (FISH) were the Spectrum Green labeled centromere of 8 (Vysis, Downers Grove, IL, USA) and the Spectrum Green labeled $2 \mathrm{~kb}$ sized MADS-IX sequence. Primers were designed from the BAC clone (NCBI, NIH, USA, RP11-407) containing the MADS-IX sequence to obtain a $2 \mathrm{~kb}$ sized PCR product encompassing the MADS-IX region (Table 1).

The $2 \mathrm{~kb}$ DNA fragment was run on a $1 \%$ gel, isolated from gel and eluted in water, and was labeled with Spectrum Green using the nick translation method, following the manufacturer's instructions (Vysis). Three-day-old slides were denatured in $70 \%$ formamide $/ 2 \times$ SSC (sodium citrate/chloride) solution at $74^{\circ} \mathrm{C}$. The dehydrated and air-dried slides were used for hybridization according to published methods with some modifications $[23,24]$. The slides were counter-stained with 4,6-diamidino-2phenylindole dihydrochloride (Molecular Probes, Eugene, OR, USA) and images were captured on the Nikon E800 using the Quips Pathvysion system (Applied Imaging,

\section{Results}

\section{Isolation of candidate MADS}

RDA was performed with the DNA recovered by SCM from normal cells and from metastatic cells in the lymph nodes of a patient with ductal mammary carcinoma. Since the objective was to isolate sequences that are lost in the progression of cells from normal to metastasis, we focused on the products of RDA in which the metastatic cell DNA was used as the driver and the normal cell DNA was the tester.

As shown in Fig. 1, RDA differential products were isolated and DNA was extracted from each of the five bands obtained from the loss side of the third round of hybridization, and DNA from each band was cloned separately. One hundred clones from each DNA band were saved and a subset of 100 clones (20 clones from each differential product) was selected randomly for further characterization. These 100 clones were probed (labeled with $\alpha^{32} P$-dCTP) on dot blots containing normal cell DNA and metastatic cell DNA. It was found that 79 clones hybridized only with the normal cell DNA and did not hybridize with DNA from the metastatic cells (Fig. 2a). The remaining clones hybridized with both normal cell DNA and the metastatic cell DNA. This may have been due to incomplete subtraction. Of the 79 clones that hybridized only with the DNA of the normal cells, 50 were selected and tested further by Southern blotting to verify the RDA results. None of these clones hybridized with the DNA of the metastatic cells, and all were detected in normal cells (Fig. 2b).

\section{Sequencing and homology search of candidate MADS}

RDA derived 50 clones that were sequenced and those with identical sequences were grouped. Ten unique candidate MADS were thus identified. The sequence homologies found by searching the nucleotide databases of these MADS are presented in Table 2. The search revealed that these MADS have homology with sequences on chromosome 1 (MADS-III), chromosome 5 (MADS-I), chromosome 6 (MADS-VII and MADS-VIII), chromosome 7 (MADS-VI), chromosome 10 (MADS-IX), chromosome 15 (MADS-X), chromosome 16 (MADS-V) and chromosome 21 (MADS-II). Nine of these MADS have about 82-99\% homology to known human DNA sequences, and the remaining MADS-IV was found to be novel, having no homology with the sequences in the gene banks.

\section{Radiation hybrid mapping of MADS}

We used the GeneBridge 4 Radiation Hybrid panel (Research Genetics, Inc) to localize these 10 MADS on human chromosomes. For this physical mapping, and also to be able to use these sequences to screen additional patient samples, primer pairs were designed for each sequence and tested by PCR on a positive control (human genomic DNA) and on two negative controls (Chinese hamster genomic DNA and no template). Of the 10 MADS 
Table 1

\begin{tabular}{lll}
\hline Primers used in representational difference analysis (RDA) and different PCR screening experiments \\
\hline Primer/product size & Primer sequence (forward) & Primer sequence (reverse) \\
\hline RDA representation & $\mathrm{R} 24,5^{\prime}$-agcactctccagcctctcaccgca-3' & $\mathrm{R} 12,5^{\prime}$-gatctgcggtga-3' \\
RDA first-round hybridization & $\mathrm{J} 24,5^{\prime}$-accgacgtcgactatccatgaaca-3' & $\mathrm{J} 12,5^{\prime}$-gatctgttcatg-3' \\
RDA second-round hybridization & $\mathrm{N} 24,5^{\prime}$-aggcaactgtgctatccgagggaa-3' & $\mathrm{N} 12,5^{\prime}$-gatcttccctcg-3' \\
RDA third-round hybridization & Same as first round (J24) & Same as first round (J12) \\
PTEN (556 bp) & $5^{\prime}$-ctcagattgccttataatagtc-3' & $5^{\prime}$-tcatgttactgctacgtaaac-3' \\
MADS-IX (180 bp) & $5^{\prime}$-aggtaggtagagtaacaggtttgttt-3' & $5^{\prime}$-gatctgttctcccttttctttagctt-3' \\
FISH fragment (2 kb) & $5^{\prime}$-actatgtatgtgagcatctgtttgc-3' & $5^{\prime}$-tgcactcttcacttgagtaaacttg-3' \\
$\beta$-Actin (295 bp) & $5^{\prime}$-tcacccacactgtgcccatctacga-3' & $5^{\prime}$-cgacgtagcacagcttctcctta-3' \\
\hline
\end{tabular}

$\mathrm{FISH}$, fluorescence in situ hybridization.

Figure 1

(a)

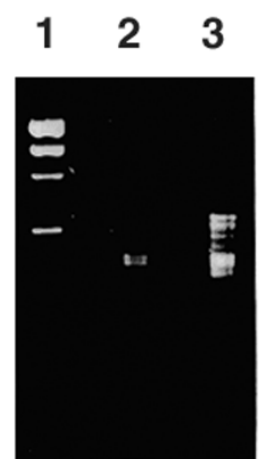

(b)

(c)
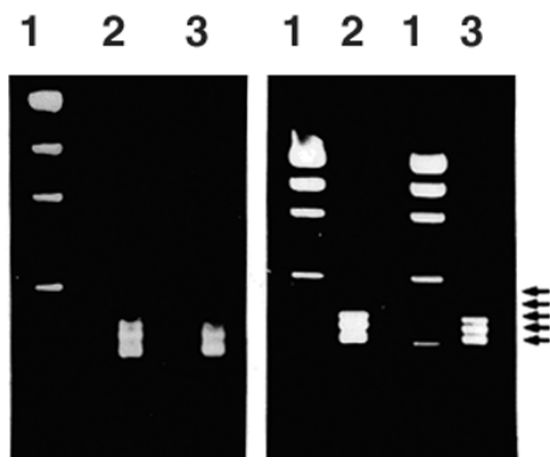

Isolation of representational difference analysis (RDA) differential products from single-cell microdissected archival breast tissues. RDA hybridization was of the normal cell DNA versus the metastatic tumor cell DNA and was performed in two ways. In the first RDA (lane 2) the metastatic cell DNA was used as the tester (which should yield differential sequences gained during malignant transformation or in the process of becoming metastatic), and in the second RDA (lane 3 ) normal DNA was used as the tester (which should yield sequences that were lost from the metastatic cells). (a), (b) and (c) Three rounds of RDA hybridizations were performed. In the third round of hybridization, the 'gain' lane contained four DNA bands (faint fourth band) ranging from 200 to $300 \mathrm{bp}$ in size (c, lane 2) whereas in the 'loss' lane there are five bands (faint fourth band) ranging from 200 to 370 bp (c, lane 3) in size (arrows). Lane 1, low molecular weight DNA marker (2kb).

tested, only five (MADS-IV, MADS-VII, MADS-VIII, MADS-IX and MADS-X) were successfully mapped to specific human chromosomes (Table 2). Of these five MADS, MADS-IV did not show any match with gene bank sequences and therefore appeared to be a novel DNA sequence. The chromosome localizations for MADS-VII, MADS-VIII, MADS-IX and MADS-X obtained by physical mapping and by homology search data were in agreement.

Figure 2

(a)

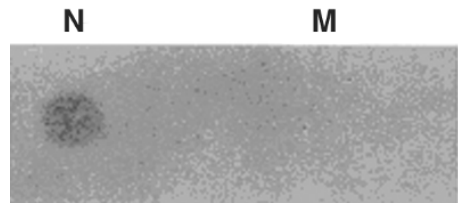

Southern blot analysis using MADS-IX as an $\alpha^{32} \mathrm{P}$-dCTP probe on normal amplicon DNA and on metastatic amplicon DNA. (a) A dot blot and (b) a Southern blot showing positive signals only in the normal cell DNA (N) but not in the metastatic cell DNA (M).

\section{Further characterization of MADS-IX}

While the homology results of MADS-IX revealed that it has $94 \%$ homology with the sequence on chromosome 10q (BAC-AC022541.10), the radiation hybrid mapping showed that MADS-IX is localized to a $21 \mathrm{cR}$ interval between markers D105539 and D10S549, corresponding to human chromosome 10 band q21.1 [25]. To confirm that MADS-IX is not an artifact and is actually a human DNA sequence located on chromosome 10q, we used the sequence as a fluorescence in situ hybridization probe on human metaphase chromosomes. Based on the sequence of the BAC clone (NCBI, RP11-407) encompassing MADS-IX, a $2 \mathrm{~kb}$ DNA fragment containing MADS-IX was generated by PCR. To localize MADS-IX cytologically, we labeled the $2 \mathrm{~kb}$ sized MADS-IX with Spectrum Green (Vysis) and hybridized on normal human metaphase chromosomes. Figure 3 shows that the MADS-IX probe was hybridized on the $10 q 21$ region. The Spectrum Green labeled centromere of chromosome 8 (Vysis) was used as a control.

To determine whether MADS-IX is indeed associated with metastasis, DNA was screened from cells recovered with the LCM method from normal, primary and metastatic tissue 
Table 2

The length, homology search and radiation hybrid mapping results of the metastasis-associated DNA sequences (MADS)

\begin{tabular}{|c|c|c|c|}
\hline MADS & Length (bp) & Homology & Radiation hybrid mapping \\
\hline MADS-I & 205 & Chromosome 5 (97\%) AC005915.1 & Not successful \\
\hline MADS-II & 144 & Chromosome 21 (99\%) AC010463.6 & Not successful \\
\hline MADS-III & 277 & Chromosome 1 (97\%) AL359265.8 & Not successful \\
\hline MADS-IV & 185 & No match & Chromosome 5; places 19.72 cR from WI-6737 \\
\hline MADS-V & 220 & Chromosome 16 (98\%) AC004234.1 & Not successful \\
\hline MADS-VI & 242 & Chromosome 7 (97\%) AC09333.1 & Not successful \\
\hline MADS-VII & 190 & Chromosome 6 (98\%) AL121935.17 & Chromosome 6; places 1.51 cR from WI-3110 \\
\hline MADS-VIII & 170 & Chromosome 6 (82\%) AC013429.12 & Chromosome 6; places 3.67 cR from AFMA191WD1 \\
\hline MADS-IX & 180 & Chromosome 10 (94\%) AC022541.10 & Chromosome 10; places 5.66 cR from D10S546 \\
\hline MADS-X & 192 & Chromosome 15 (98\%) AC104260.5 & Chromosome 15; places 1.71 cR from D15S157 \\
\hline
\end{tabular}

Figure 3

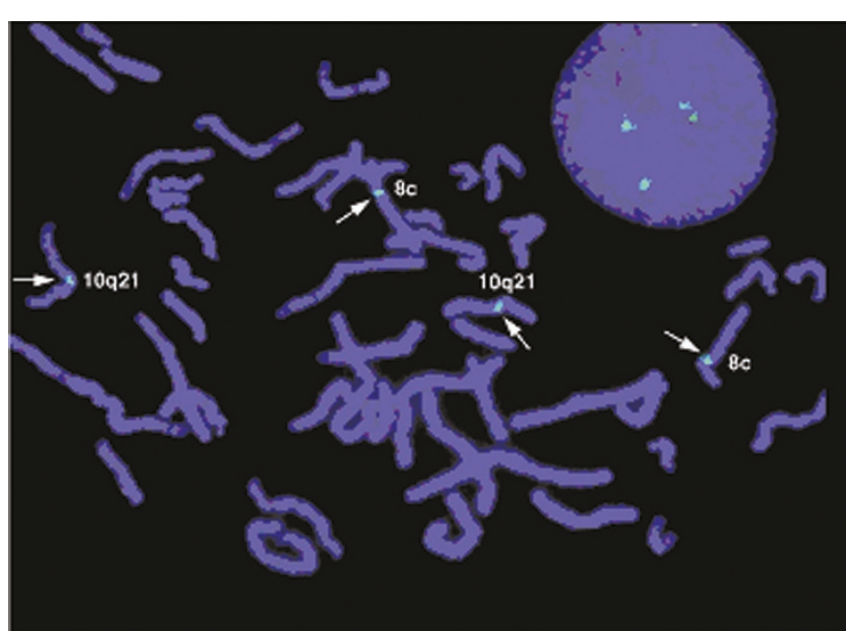

Localization of the $2 \mathrm{~kb}$ DNA fragment containing MADS-IX on human metaphase chromosomes. The centromere of chromosome 8 was used as a positive control probe. MADS-IX was found to localize around the 10q21 chromosome region (close to the centromere). Both the MADS-IX and the Spectrum Green labeled centromere of 8 were labeled with spectrum green (Vysis, Downers Grove, IL, USA), and the chromosomes were counter-stained by 4,6-diamidino-2-phenylindole dihydrochloride.

samples from five additional patients. Because MADS-IX was mapped to chromosome 10q21, we also wanted to determine whether it was located in the region encompassing a well-known metastasis-associated gene, PTEN [15].

First, DNA samples from these five patients were

\section{Figure 4}

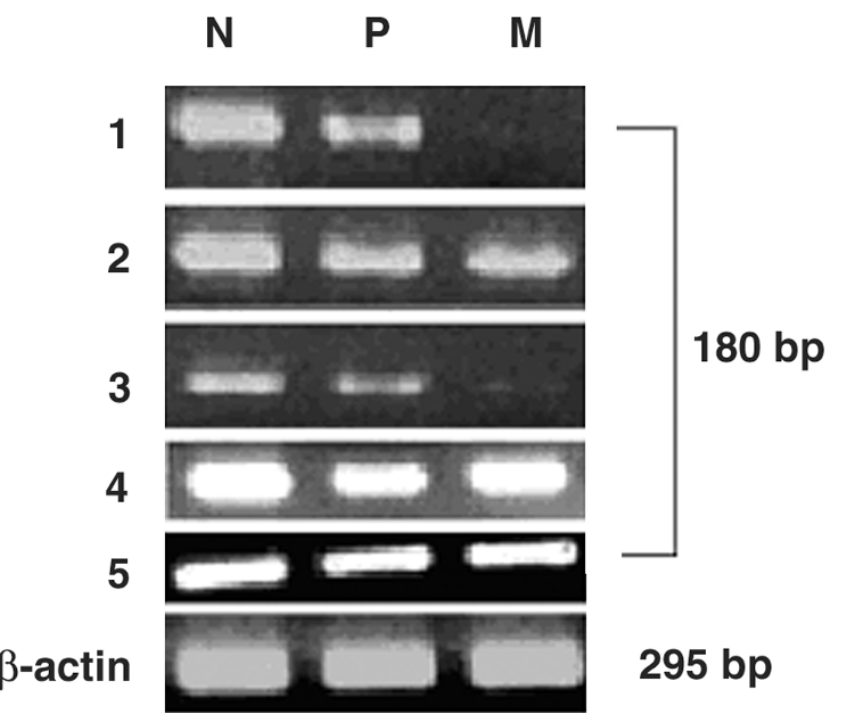

PCR screening of MADS-IX on normal cell DNA, primary cell DNA and metastatic cell DNA samples of five patients. PCR results show the target DNA band (180 bp) missing in patients 1 and 3 . While MADS-IX showed a reduction in the intensity (about one-half) of the PCR band (heterozygous loss) in the primary tumors $(P)$ of patients 1 and 3 , it is completely missing (homozygous loss) in their metastatic (M) cell DNA samples but present in normal cell DNA $(\mathrm{N})$. $\beta$-Actin was used as an internal control.

and PTEN. MADS-IX showed PCR amplification in normal cell DNA samples of all the five patients, but the intensities of the PCR bands were less in the primary tumor cell DNA samples and were totally undetectable in the metastatic cell DNA of patients 1 and 3 (Fig. 4). The PTEN gene, on the contrary, was detected, without any difference in the intensity of PCR bands in the DNA samples of normal 
Figure 5

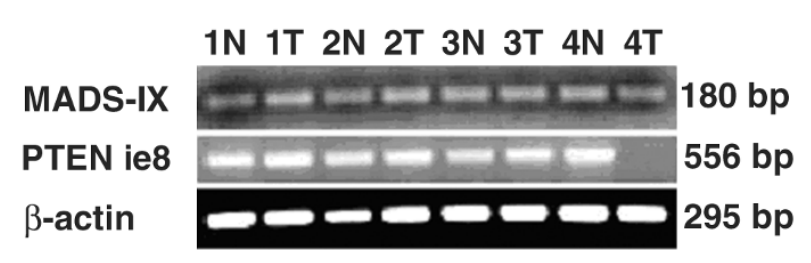

PCR screening of MADS-IX and PTEN on DNA samples from four tumor cell lines and matched normal DNA. Results (first row) show that MADS-IX is present in all the tumor cell lines (HCC-1806, HCC-1143, HCC-1428 and HCC-1937) especially the HCC-1937 cell line, which has loss of a region encompassing the PTEN gene. Screening of PTEN in these four cell lines (second row) show its presence in the HCC-1806, HCC-1143 and HCC-1428 tumor cell lines but, as expected, it is missing in $\mathrm{HCC}-1937$. $\beta$-Actin was used as an internal control.

cells, primary tumor cells and metastatic lymph node cells of all five patients.

Second, MADS-IX and PTEN were screened in four breast tumor cell lines (HCC-1806, HCC-1143, HCC-1428 and HCC-1937) and matched normal DNA. The first three tumor cell lines had known losses in specific chromosomal regions, and the fourth had loss of homozygosity of the PTEN gene. PCR screening of MADS-IX and PTEN showed that MADS-IX is present in all four tumor cell lines, but PTEN is only present in cell lines HCC-1806, HCC-1143 and HCC-1428 and is missing in HCC-1937 (Fig. 5).

\section{Discussion}

Reports show a statistically significant increase in loss of heterozygosity events involving unknown genes on chromosome 16q [26], on chromosome 10q [15] and on chromosome $14 q$ [27], in metastatic breast cancers. Measuring loss of heterozygosity, O'Connell et al. [27] found that the majority of lymph node-negative primary breast tumors did not amplify a region linked to D14S62 and D14S51, while lymph node-positive breast tumors retained heterozygosity for these markers. These data could imply the existence of metastasis promoting gene(s) in that region or, alternatively, the observed molecular changes may be used as a marker of metastatic propensity [28].

In the present investigation we used RDA in an attempt to identify genetic alterations related to breast cancer metastasis by comparing normal cell DNA and metastatic cell DNA of a ductal breast carcinoma patient. Ten unique candidate MADS were identified and the sequence homologies found by searching the gene bank of these MADS are presented in Table 2. The search revealed 82-99\% homology to known human gene sequences for nine of these MADS but MADS-IV was found to be novel, having no homology with the sequences in the gene banks. Further characterization of MADS-IX revealed interesting information on the genes and sequence tagged sites (STS) markers surrounding it. Within a range of 1.4 Mb of MADS-IX, we noticed two CDC2 genes (cell division cycle 2 protein, isoform 1 and isoform 2) and two splice variants of the ZWINT gene (without known exact function). In the range of $821 \mathrm{~kb}$, there are nine STS markers (RH26942, SHGC-58267, SHGC-79901, AFM336XD1, SHGC-103705, SHGC-3856, SHGC-84937, STSG72171 and SHGC-81245).

Physical and cytological mapping of MADS-IX confirmed that it is located at 10q21.1. Screening of MADS-IX on DNA samples from five more sets of normal, primary and matched metastatic tumor samples (Fig.4) revealed that MADS-IX is lost in the transition from normal cells to primary cells to metastasis cells in two of five cases (three of six cases if the case used in the RDA assay is included). PCR screening of PTEN on these five patient samples and screening of MADS-IX and PTEN on a breast carcinoma cell line that had loss of homozygosity of the PTEN gene revealed that MADS-IX is neither a part of the PTEN gene or localized in the homozygous loss region of chromosome $10 \mathrm{q}$ encompassing the PTEN gene. These findings together strongly suggest that MADS-IX is a candidate marker for a novel metastasis suppressor gene. Additional archival cases are being screened to determine whether MADS-IX is a reliable marker for metastasis in ductal breast cancers.

\section{Competing interests}

None declared.

\section{Acknowledgments}

The authors are thankful to Dr Z Zhuang and Dr MR Emmert-Buck of the National Cancer Institute/National Institutes for Health for training PMRA in laser capture microdissection, and to Dr R Kucherlapati of the Department of Molecular Genetics for allowing PMRA to use his laboratory facilities at Albert Einstein College of Medicine for radiation hybrid mapping work. Funds from the US Army (DAMD17-99-1-9055) and Klingers' Research Foundation, New York, USA to PMRA are gratefully acknowledged.

\section{References}

1. Lewis BJ, Conry RM: Breast cancer. In Cecil Textbook of Medicine. Edited by Bennett J, Plum F. USA: WB Saunders: 1992:1320-1325.

2. Scorilas A, Yotis J, Gouriotis D, Keramopoulos A, Ampela K Trangas T, Talieri M: Cathepsin-D and C-erb-B 2 have an additive progostic value for breast cancer patients. Anticancer Res 1993, 13:1895-1900.

3. Fisher B, Dignam J, Wolmark N, DeCillis A, Emir B, Wickerham DL, Bryant J, Dimitrov NV, Abramson N, Atkins JN, Shibata H, Deschenes L, Margolese RG: Tamoxifen and chemotherapy for lymph node-negative, estrogen receptor-positive breast cancer. J Natl Cancer Inst 1997, 89:1673-1682.

4. Callahan R: MMTV-induced mutations in mouse mammary tumors: their potential relevance to human breast cancer. Breast Cancer Res Treat 1996, 39:33-44.

5. Pignatelli M, Vessey CJ: Adhesion molecules: novel molecular tools in tumor pathology. Hum Pathol 1994, 25:849-856.

6. Shi YE, Liu YE, Lippman ME, Dickson RB: Progestins and antiprogestins in mammary tumour growth and metastasis. Hum Reprod 1994, 9:162-173. 
7. Tozawa K, Sakurada S, Kohri K, Okmoto T: Effect of anti-nuclear factor $k$ B reagents in blocking adhesion of human cancer cell to vascular endothelial cells. Cancer Res 1995, 55:4162-4167.

8. Bashyam MD: Understanding cancer metastasis: an urgent need for using differential gene expression analysis. Cancer 2002, 94:1821-1829.

9. Steeg PS, de la Rosa A, Flatow U, MacDonald NJ, Benedict M, Leone A: Nm23 and breast cancer metastasis. Breast Cancer Res Treat 1993, 25:175-187.

10. Freije JM, MacDonald NJ, Steeg PS: Nm23 and tumour metastasis: basic and translational advances. Biochem Soc Symp 1998, 63:261-271.

11. Phillips KK, White AE, Hicks DJ, Welch DR, Barrett JC, Wei LL, Weissman BE: Correlation between reduction of metastasis in the MDA-MB-435 model system and increased expression of the Kai-1 protein. Mol Carcinogen 1998, 21:111-120.

12. Yang $X$, Wei L, Tang C, Slack R, Montgomery E, Lippman M: KAl1 protein is down-regulated during the progression of human breast cancer. Clin Cancer Res 2000, 6:3424-3429.

13. Toh Y, Pencil SD, Nicolson GL: Analysis of the complete sequence of the novel metastasis-associated candidate gene, mta1, differentially expressed in mammary adenocarcinoma and breast cancer cell lines. Gene 1995, 159:149-154.

14. Lee JH, Welch DR: Identification of highly expressed genes in metastasis-suppressed chromosome 6/human malignant melanoma hybrid cells using subtractive hybridization and differential display. Int J Cancer 1997, 71:1035-1044.

15. Li J, Yen C, Liaw D, Podsypanina K, Bose S, Wang SI, Puc J, Miliaresis C, Rodgers L, McCombie R, Bigner SH, Giovanella BC, Ittmann M, Tycko B, Hibshoosh H, Wigler MH, Parsons R: PTEN, a putative protein tyrosine phosphatase gene mutated in human brain, breast, and prostate cancer. Science 1997, 275:1943-1947.

16. Steck PA, Pershouse MA, Jasser SA, Yung WK, Lin H, Ligon AH, Langford LA, Baumgard ML, Hattier T, Davis T, Frye C, Hu R, Swedlund B, Teng DH, Tavtigian SV: Identification of a candidate tumour suppressor gene, MMAC1, at chromosome $10 q 23.3$ that is mutated in multiple advanced cancers. Nat Genet 1997, 15:356-362.

17. Seraj MJ, Samant RS, Verderame MF, Welch DR: Functional evidence for a novel human breast carcinoma metastasis suppressor, BRMS1, encoded at chromosome 11q13. Cancer Res 2000, 60:2764-2769.

18. Lisitsyn NA, Lisitsyn NM, Wigler M: Cloning the differences between two complex genomes. Science 1993, 259:951-964.

19. Mukherjee B, Zhao H, Parashar B, Sood BM, Mahadevia PS, Klinger HP, Vikram B, Achary PMR: Microsatellite dinucleotide (T-G) repeat: a candidate marker for breast metastasis. Cancer Detect Prevent 2003, 68:1-5.

20. Emmert-Buck MR, Bonner RF, Smith PD, Chuaqui RF, Zhuang Z, Goldstein SR, Weiss RA, Liotta LA: Laser capture microdissection. Science 1996, 274:998-1001.

21. Lisitsyn NA, Lisitsina NM, Dalbagni G, Barker $P$, Sanchez CA, Gnarra J, Linehan WM, Reid BJ, Wigler MH: Comparative genomic analysis of tumors: detection of DNA losses and amplification. Proc Natl Acad Sci USA 1995, 92:151-155.

22. http://www-genome.wi.mit.edu/cgi-bin/contig/rhmapper

23. Mathew S, Murty VV, Hunziker W, Chaganti RS: Subregional mapping of 13 single-copy genes on the long arm of chromosome 12 by fluorescence in situ hybridization. Genomics 1992, 14:775-779.

24. Rao PH, Murty VVVS, Gaidano G, Hauptschein R, Dalla-Favera R, Chaganti RSK: Subregional localization of 20 single-copy loci to chromosome 6 by fluorescence in situ hybridization. Genomics 1993, 16:426-430.

25. http://ncbi.nlm.nih.gov

26. Lindblom A, Rotstein S, Skoog L, Nordenskjold M, Larsson C: Deletions of chromosome 16 in primary familial breast carcinomas are associated with development of distant metastases. Cancer Res 1993, 53:3707-3711.

27. O'Connell P, Fischbach K, Hilsenbeck S, Mohsin SK, Fuqua SA, Clark GM, Osborne CK, Allred DC: Loss of heterozygosity at D14S62 and metastatic potential of breast cancer. J Natl Cancer Inst 1999, 91:1391-1397.

28. Welch DR, Rinker-Schaeffer CW: What defines a useful marker of metastasis in human cancer? J Natl Cancer Inst 1999, 91:1351-1353.

\section{Correspondence}

Patnala Mohan R Achary, Metastasis Laboratory, Ullmann Building1219, Department of Radiation Oncology, Albert Einstein College of Medicine, 1300 Morris Park Avenue, Bronx, NY 10461, USA. Tel: +1 718430 2699; fax: +1 718430 2454; e-mail: achary@aecom.yu.edu 